\title{
BMJ Open Self-referral psychological treatment centre for young adults: a 2-year observational evaluation of routine practice before and after treatment
}

\author{
Karin Halje, ${ }^{1}$ Toomas Timpka, ${ }^{2,3}$ Petra Tylestedt, ${ }^{1}$ Anna-Karin Adler, ${ }^{1}$ \\ Lena Fröberg, ${ }^{1}$ Tommy Schyman, ${ }^{2}$ Kristoffer Johansson, ${ }^{2}$ Katarina Dahl ${ }^{1}$
}

To cite: Halje K, Timpka T, Tylestedt $P$, et al. Self-referral psychological treatment centre for young adults: a 2year observational evaluation of routine practice before and after treatment. BMJ Open 2015:5:e008030.

doi:10.1136/bmjopen-2015008030

- Prepublication history for this paper is available online. To view these files please visit the journal online (http://dx.doi.org/10.1136/ bmjopen-2015-008030)

Received 23 February 2015 Revised 2 July 2015 Accepted 30 July 2015

\section{(1) CrossMark}

${ }^{1}$ Young Adults Centre, Region Östergötland, Linköping, Sweden

${ }^{2}$ Department of Medical and Health Sciences, Linköping University, Linköping, Sweden

${ }^{3}$ Unit for Health Analysis, Centre for Healthcare Development, Region Östergötland, Linköping, Sweden

Correspondence to Dr Toomas Timpka; toomas.timpka@liu.se

\section{ABSTRACT}

Objectives: To examine a self-referral psychological service provided to young adults with regard to effects on anxiety, depression and psychological distress and to explore client factors predicting non-adherence and non-response.

Design: Observational study over a 2-year period.

Setting: Young Adults Centre providing psychological services by self-referral (preprimary care) to Linköping, Åtvidaberg, and Kinda municipalities (combined population 145000 ) in Östergötland county, Sweden.

Participants: 607 young adults (16-25 years of age); $71 \%$ females $(n=429)$.

Intervention: Individually scheduled cognitive behavioural therapy delivered in up to six $45 \mathrm{~min}$ sessions structured according to an assessment of the client's mental health problems: anxiety, depression, anxiety and depression combined, or decreased distress without specific anxiety or depression.

Primary outcome measures: Pre-post intervention changes in psychological distress (General Health Questionnaire-12, GHQ-12), Hospital Anxiety and Depression Scale Anxiety/Depression (HADS-A/D).

Results: 192 clients (32.5\%) discontinued the intervention on their own initiative and 39 clients $(6.6 \%)$ were referred to a psychiatric clinic during the course of the intervention. Intention-to-treat analyses including all clients showed a medium treatment effect size $(\mathrm{d}=0.64)$ with regard to psychological distress, and small effect sizes were observed with regard to anxiety $(d=0.58)$ and depression ( $d=0.57)$. Restricting the analyses to clients who adhered to the agreed programme, a large effect size $(\mathrm{d}=1.26)$ was observed with regard to psychological distress, and medium effect sizes were observed with regard to anxiety $(d=1.18)$ and depression $(d=1.19)$. Lower age and a high initial HADS-A score were the strongest risk factors for non-adherence, and inability to concentrate and thinking of oneself as a worthless person increased the risk for discontinuation.

Conclusions: We conclude that provision of psychological services to young people through a self-referral centre has potential to improve long-term mental health in communities, but management of non-adherence remains a central challenge.

\section{Strengths and limitations of the study}

- This observational study of a self-referral psychological service for young adults was performed in a routine setting and the data set involved all clients admitted during a 2-year period.

- The main analyses were performed using an intention-to-treat (ITT) approach to structuring of data, based on that evaluations using the ITT and completer approaches in parallel have been called for when reporting from routine clinical psychological interventions.

- The observational study design cannot ascertain what proportion of the recovery rates can be accounted for by factors such as spontaneous remission over time.

- Although the self-report measures, the Hospital Anxiety and Depression Scale Anxiety/ Depression (HADS-A/D) and General Health Questionnaire-12, have solid psychometric properties and the cut points used are those reported as being scores with an appropriate balance of diagnostic specificity and sensitivity, they did not formally diagnose the clients.

\section{INTRODUCTION}

Mental disorders account for a large proportion of the global disease burden in young people. ${ }^{1}$ Poor mental health is also associated with other health and development issues in youth, for example, lower educational achievements, substance abuse, violence and poor sexual health. ${ }^{2}$ At the global level, key challenges to addressing the mental health needs of the young include the low capacity to provide quality mental health services to young people and the stigma associated with mental disorders. ${ }^{3}$ It has been proposed that the high prevalence of mental illnesses such as depression and anxiety in the general population should be addressed through psychological treatment 
centres, ${ }^{4}$ and recently, several evaluations of easily accessible psychological services integrated within primary care have been published. ${ }^{5}$ For instance, from studies performed in association to the large-scale Improving Access to Psychological Therapies (IAPT) initiative within the UK National Health Service, it has been reported that $55-56 \%$ of patients who had attended more than twice could be classified as recovered (scoring below the clinical cut-off on self-report measures assessing depression and anxiety ${ }^{6}$ with corresponding uncontrolled effect sizes in the 1.0-1.1 range for anxiety and depression. ${ }^{7}$

Easily accessible mental health services have also been developed for adolescents and young adults. The Australian headspace is an extensive programme addressing young people 12-25 years of age. ${ }^{8}$ The programme involved 55 centres in 2014 and the plan is to scale up to 100 centres in $2016 .{ }^{9}{ }^{10}$ Recently, intervention outcomes with regard to psychological distress and social functioning have been reported from this initiative. ${ }^{11}$ The adolescent and young adult context is associated with specific challenges for psychological service provision. For instance, low adherence has been reported as a key problem when treating young clients in community settings. ${ }^{12}{ }^{13}$ A psychological intervention requires a substantial commitment from clients in terms of attending therapy sessions, engaging with a therapist, and often completing homework between sessions. As nonadherence directly influences service effectiveness and delivery costs, ${ }^{14}$ identification of the factors associated with programme discontinuation is vital when developing mental health services for this age group. We therefore set out to examine the prospective outcomes of a self-referral psychological service provided to young adults over a 2-year period in Östergötland County, Sweden. Specifically, the aims of this study were to assess effects on anxiety, depression and psychological distress and explore client factors that predict non-adherence and non-response to the intervention.

\section{BACKGROUND}

In Östergötland County, Sweden (population 440 000), a survey of the county residents' mental health status in 2006 revealed that young adults (18-29 years of age) were affected by mental conditions to a larger extent than other age groups. ${ }^{15}$ Several other counties reported similar findings, ${ }^{16}$ and in June 2007, the Swedish government allocated SEK 214 million (US $\$ 70$ million) to be divided among county councils providing services for young people with poor mental health. In the same year, the Unga Vuxna (Young Adults) service was initiated in Östergötland County. Methods and theoretical perspectives from cognitive-behavioural therapy $(\mathrm{CBT})^{17}$ were used as one starting point for the service design. Rather than diagnosing and treating psychiatric disease, the goal was to be able to help young people with psychological problems to learn strategies to manage undesired thoughts and behaviours associated with anxiety, depression and psychological distress. The policy-level rationale for this strategy was that most mental disorders begin during youth, although they are often first detected later in life. ${ }^{3}$ Therefore, preventing long-term mental health problems by provision of psychological services to young adults without stigmatising them with psychiatric diagnoses was an explicit objective of the programme. The service was to be provided only by psychotherapists with no physicians or nurses associated with it. Another starting point was that the most frequent mental health problems among youth, depression and anxiety, share central characteristics common to emotional disorders. ${ }^{18-20}$ In addition, preventive measures directed at depression and anxiety can be integrated, because the disorders have a number of modifiable predisposing factors in common, for example, general distress, negative affect and negative cognition. ${ }^{21} \mathrm{~A}$ pragmatic transdiagnostic perspective ${ }^{22}$ was therefore applied in the design of the intervention programme.

\section{METHODS}

An observational, prospective, cohort design was used for the study. Clients who were assessed at the Young Adults Centre were asked to complete standardised measures of psychological distress, depression and anxiety before and after treatment. The data were collected in 2008 and 2009.

\section{Ethics statement}

This study is considered a routine service evaluation, that is, it was based on routine databases established for the purpose of systematically and continuously developing and securing the quality of psychological services. The study did therefore not fall under the definition of research according to Swedish legislation (SFS 2008:355) because we did not manipulate clinicians' treatment decisions or use experimental interventions. Accordingly, no approval from an ethics committee was required for the study. All clients were as a part of the routines at the clinic informed verbally and in writing about that their data may be used for analyses of service quality assurance and their verbal consent were asked for. Written consent was not requested due to all clients being asked to fill in self-report forms for collection of diagnostic data. The same data were used for service quality control. Non-adherence with this procedure was noted in the electronic client record. No informed consent from the next of kin, caretakers or guardians on behalf of the minors included in the service quality evaluation was obtained. Minors older than 15 years of age were in accordance with established practices in Swedish healthcare assumed to make own decisions regarding their care and the associated quality assessment. The age was determined by the upper age limit for paediatric care in Sweden. Personal identification data were removed from the records (all information 
was de-identified) prior to that the data set being received by the authors for statistical analyses. The evaluation design was confirmed by consultation with the Ethics Committee at Linköping University before initiation of the data collection.

\section{Study setting}

The Young Adults Centre provides psychological services to young adults (16-25 years of age) in Linköping, Åtvidaberg, and Kinda municipalities (combined population 145000) in Östergötland County, Sweden. Östergötland County consists of 13 municipalities, of which two (Linköping and Norrköping) account for about two-thirds of its population. A European highway and the main train connection between Stockholm and Copenhagen run across the county, which, outside urbanised areas, consists mainly of farmland. Employees at several large companies and one university situated in the county use two local airports for business travel to international destinations. The demography in Östergötland is similar to that of non-metropolitan Sweden. However, the municipalities of Östergötland tend to have a higher share of young people and fewer residents born abroad than the municipalities in other non-metropolitan counties.

The Young Adults Centre employs four psychotherapists with at least 1 year of postgraduate specialist training in CBT. Self-referral by telephone is the only means for young adults to contact the centre. Preliminary admission to the service is regulated by therapist triage by telephone. At the time of the study, the psychotherapeutic programme at the centre consisted of interventions customised into four threads based on the client's problems; thread 1 was adapted for anxiety cases, thread 2 for depression cases, thread 3 for clients assessed as both anxiety and depression cases and thread 4 for psychological distress cases without specific anxiety or depression. The programme toolbox contained bestpractice CBT methods, for example, psychoeducation, exposure and cognitive restructuring. ${ }^{23-25}$ The clinical intervention procedure comprised of six sessions, each lasting $45 \mathrm{~min}$. In the first session, the nature of the client's problems was evaluated clinically and using selfreport forms for psychological distress (General Health Questionnaire (GHQ)-12), ${ }^{26}$ Hospital Anxiety and Depression Scale Anxiety/Depression (HADS-A/D). ${ }^{27}$ Following the evaluation, clients analysed to have minor problems (based on clinical valuation and below cutpoint self-report scores) were offered self-help instructions within the first session, while the remaining clients were offered a customised intervention programme in the thread suggested by the evaluation outcomes. For each client, an individual treatment plan was collaboratively decided. In the concluding part of the first session and during the second session, the client's problems were analysed in detail, psychoeducation initiated and homework assigned. The content of sessions 3-5 followed the jointly decided treatment plan. Homework assignments were followed-up and new tasks assigned based on the session contents. At the last session, a personalised postintervention knowledge and skills maintenance programme was outlined to be used after discharge and a summary of the intervention outcome was made together with the client. The final session and intervention was ended with that the client independently scored the three self-report forms.

A report on the client's progress, including whether the client had expressed any suicidal intent, was after each session documented in the electronic client record. Clients received whatever psychological treatment their therapist prescribed in parallel with other ongoing interventions, for example, provision of practical or social help at the employment office, and further medical investigations. Clients also continued to receive pharmacotherapy if previously prescribed by their physician.

\section{Data collection}

Instruments assessing general psychological distress (GHQ-12), anxiety (HADS-A) and depression (HADS-D) were administered as a part of the service at the Young Adults Centre on two occasions: before treatment and when the client had completed their programme. The 12-item version of the GHQ has demonstrated validity with young adults $(17+$ years $){ }^{26}$ The sensitivity and specificity for both HADS-A and HADS-D are similar to the sensitivity and specificity achieved by GHQ-12 and they perform well in assessing the symptom severity of anxiety disorders and depression in somatic, psychiatric and primary care clients and in the general population. ${ }^{27}$ Data on adherence are recorded in two dimensions by the therapists after the last session, that is, whether the client has discontinued the CBT programme on their own initiative or has been discontinued from the programme due to referral to a psychiatric clinic.

\section{Participants}

To be included, clients were required to have an initial assessment at the Young Adults Centre between 1 January 2008 and 31 December 2009.

\section{Data analyses}

The data were first exhibited using descriptive statistical methods. In accordance with the structure of the intervention programme, cases were for this analysis defined as follows: an anxiety case was a client with a HADS-A score $>7$, a depression case a client scoring HADS-D $>7$, and a psychological distress case a client scoring HADS-A $<8$ and HADS-D $<8$, while GHQ-12 was scored 3 or more. Recovery (treatment response) was defined as scoring below the cut-off point for all three self-report instruments. In the second step, treatment effect sizes (Cohen's d) were computed to quantify treatment effects with regard to anxiety (before and after treatment difference in HADS-A scores), depression 


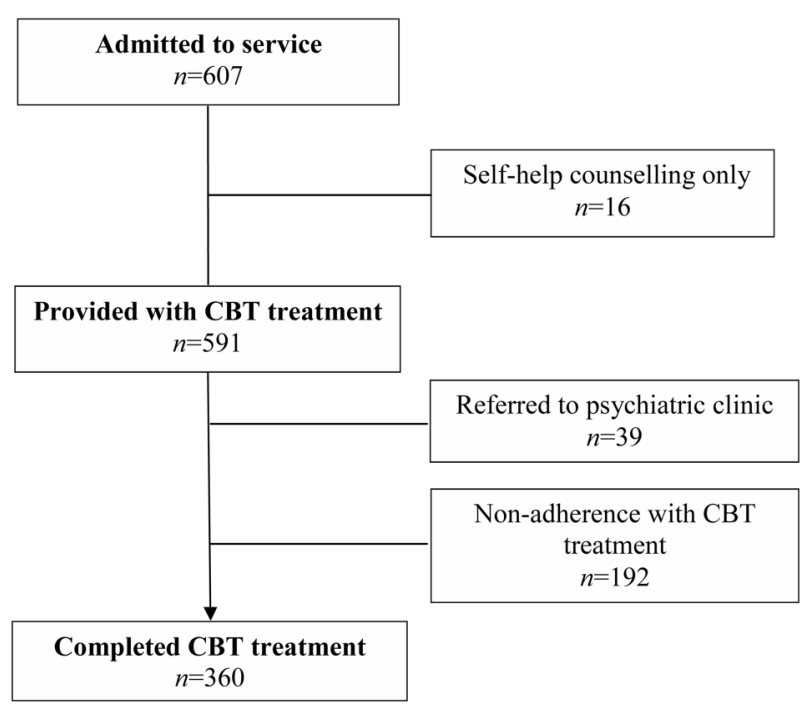

Figure 1 Study flow chart.

(differences in HADS-D scores) and psychological distress (differences in GHQ-12 scores). Effect sizes create a generally interpretable description of the size of a treatment effect. Observed values for Cohen's $d$ higher than 0.2 were interpreted as small, higher than 0.6 as medium and higher than 1.2 as large effect sizes. ${ }^{28}$ In addition, before and after treatment differences in the self-evaluation scores were established using paired $\mathrm{t}$ tests (significance level 5\%). Separate calculations were performed for the intention-to-treat (ITT) and completed-treatment approaches to structure client data for the analyses. Step-wise multiple logistic regression modelling was then used to explore client variables that predict non-response to treatment and non-adherence with the intervention. Clients who just needed one session or were referred to a psychiatric clinic were excluded from these analyses. The non-response model estimated the value of using the patient's self-assessments at baseline for prediction of persisting case status with regard to anxiety, depression or psychological distress at the follow-up visit. The response variable was scoring above the case status level at any of the self-evaluation instruments at follow-up visits (yes/no); explanatory variables were sex, age, total scores or HADS-A and HADS-D, and scores from each separate item of the GHQ-12 recorded at the first visit. In the non-adherence model, the response variable was adherence with the intervention programme as agreed with the therapist at its initiation (yes/no); the explanatory variables were sex, age, total scores or HADS-A and HADS-D, and scores from each separate item of the GHQ-12 recorded at the first visit. ORs with a 90\% CI were computed as well as p values (significance level 10\%).

To assess model performance, Hosmer and Lemeshow's goodness-of-fit test was computed for both multiple logistic regression models. In addition, pairwise correlations were computed for all explanatory variables used in the analyses to assess the occurrence of multicolinearity in the data. ${ }^{29}$

\section{RESULTS}

Six hundred-seven clients were admitted to the programme during the study period (figure 1). Seventy-one per cent $(n=429)$ of the clients were female; the median age was 23 years. An overview of the psychological case status of the client population at the time of the first session at the centre is presented in table 1 . More than four clients out of five scored above the breakpoint for anxiety case status and about every second client above the breakpoint for depression case status.

\section{Intervention effect sizes}

After the evaluation of the problem, 16 clients (2.6\%) received only one session focusing on self-help instructions and were then discharged from the programme; 8 of these clients were female $(1.9 \%$ of all female clients) and 8 were male $(4.5 \%$ of all male clients). The remaining 591 clients were referred to the psychological intervention. The ITT analysis including all clients, disregarding the treatment strategy and whether they followed the agreed treatment programme or not, showed a medium before and after treatment effect size $(\mathrm{d}=0.64)$ with regard to general distress (GHQ-12); small effect sizes were observed with regard to anxiety (HADS-A; $\mathrm{d}=0.58$ ) and depression (HADS-D; $\mathrm{d}=0.57$; table 2). Considering the clients in the respective case type categories separately, a medium effect size was observed for all three categories of clients. The beforeafter difference in treatment scores was statistically significant for all psychological problem categories and within each case status subgroup $(\mathrm{p}<0.001)$. Restricting the analyses only to clients who adhered to the agreed

Table 1 Psychological case status of the client population at the time of the first session

\begin{tabular}{|c|c|c|c|c|c|c|}
\hline \multirow[b]{2}{*}{ Case status } & \multicolumn{2}{|c|}{ Female $(n=429)$} & \multicolumn{2}{|c|}{ Male $(n=178)$} & \multicolumn{2}{|c|}{ Total $(n=607)$} \\
\hline & $\mathrm{n}$ & Per cent & n & Per cent & $\mathbf{n}$ & Per cent \\
\hline Anxiety (HADS-A) & 370 & 86.2 & 143 & 80.3 & 513 & 84.5 \\
\hline Depression (HADS-D) & 200 & 46.6 & 88 & 49.4 & 288 & 47.4 \\
\hline Psychological distress (GHQ-12) & 30 & 6.3 & 15 & 8.4 & 45 & 6.9 \\
\hline
\end{tabular}

Anxiety and depression cases were clients scoring above the cut-points for HADS-A and HADS-D, respectively. A psychological distress case was a client scoring above the cut-point for GHQ-12 and below the cut-points for HADS-A and HADS-D.

GHQ-12, General Health Questionnaire-12; HADS-A/D, Hospital Anxiety and Depression Scale Anxiety/Depression. 
Table 2 Treatment effect sizes for anxiety, depression and psychological distress according to intention-to-treat analyses $(\mathrm{n}=607)$

\begin{tabular}{|c|c|c|c|c|c|}
\hline & \multirow[b]{2}{*}{$\mathbf{n}$} & \multicolumn{2}{|l|}{ Mean score } & \multirow[b]{2}{*}{ SD (post-treatment) } & \multirow[b]{2}{*}{ Effect size (Cohen's d) ${ }^{\star}$} \\
\hline & & Pretreatment & Post-treatment & & \\
\hline \multicolumn{6}{|c|}{ Anxiety (HADS-A) } \\
\hline All & 607 & 12.20 & 9.36 & 4.86 & 0.58 \\
\hline Cases & 513 & 13.43 & 10.23 & 4.71 & 0.68 \\
\hline \multicolumn{6}{|c|}{ Depression (HADS-D) } \\
\hline All & 607 & 7.47 & 5.02 & 4.38 & 0.56 \\
\hline Cases & 288 & 10.97 & 7.36 & 4.88 & 0.74 \\
\hline \multicolumn{6}{|c|}{ Psychological distress (GHQ-12) } \\
\hline All & 604 & 6.51 & 4.12 & 3.74 & 0.64 \\
\hline Cases & 42 & 4.24 & 2.50 & 2.35 & 0.74 \\
\hline
\end{tabular}

programme, a large effect size $(\mathrm{d}=1.26)$ was observed with regard to general distress (GHQ-12); medium effect sizes were observed with regard to anxiety (HADS-A; $\mathrm{d}=1.18$ ) and depression (HADS-D; $\mathrm{d}=1.19$; table 3). Regarding the respective case type categories separately, a large effect size was observed for anxiety (HADS-A; $\mathrm{d}=1.36$ ) and depression (HADS-D; $\mathrm{d}=1.48$ ), and a medium effect size for the general distress group $(\mathrm{GHQ}-12 ; \mathrm{d}=1.3)$. The before-after difference in treatment scores was statistically significant for all psychological problem categories and within each case status subgroup $(\mathrm{p}<0.001)$.

For 403 clients (66\%) admitted to the programme, the final self-evaluation score recorded during the intervention programme persisted above the breakpoint for case status for at least one of the instruments used for quality assurance at the centre; 369 clients (61\%) scored above the breakpoint for anxiety, $164(27 \%)$ for depression, and $24(4 \%)$ for psychological distress. However, the final score was recorded in less than two-thirds of clients $(60.9 \%)$ after the agreed treatment had been completed.

\section{Programme discontinuation}

About one-third of the clients (32.5\%) discontinued the intervention on their own initiative; about 1 client in $20(6.6 \%)$ was referred to a psychiatric clinic during the course of the intervention (table 4). Low age and a high HADS-A score recorded at the first visit were the strongest risk factors for non-adherence (table 5), but reporting not being able to concentrate and thinking of oneself as a worthless person at the first visit were also found to increase the risk for discontinuation.

Among the 360 clients who completed the CBT-based intervention, the characteristics recorded at the first visit that were found to increase the risk for non-response to treatment were, most notably, self-evaluation of being unhappy and depressed and not being able to enjoy normal day-to-day activities (table 6). As expected, highinitial scores on the HADS-A and HADS-D instruments were associated with persistent case status after treatment. More surprisingly, client reports of not being constantly under strain and being capable of making

Table 3 Treatment effect sizes for anxiety, depression and psychological distress restricted to clients who completed the CBT treatment $(n=360)$

\begin{tabular}{|c|c|c|c|c|c|}
\hline & \multirow[b]{2}{*}{$\mathbf{n}$} & \multicolumn{2}{|l|}{ Mean score } & \multirow[b]{2}{*}{ SD (post-treatment) } & \multirow[b]{2}{*}{ Effect size (Cohen's d)^ } \\
\hline & & Pretreatment & Post-treatment & & \\
\hline \multicolumn{6}{|c|}{ Anxiety (HADS-A) } \\
\hline All & 360 & 11.99 & 7.22 & 4.05 & 1.18 \\
\hline Cases & 300 & 13.31 & 7.82 & 4.01 & 1.36 \\
\hline \multicolumn{6}{|c|}{ Depression (HADS-D) } \\
\hline All & 360 & 7.30 & 3.17 & 3.47 & 1.19 \\
\hline Cases & 168 & 10.83 & 4.66 & 4.18 & 1.48 \\
\hline \multicolumn{6}{|c|}{ Psychological distress (GHQ-12) } \\
\hline All & 360 & 6.47 & 2.45 & 3.17 & 1.27 \\
\hline Cases & 28 & 4.25 & 1.64 & 2.31 & 1.13 \\
\hline
\end{tabular}


Table 4 Treatment adherence status for clients provided with CBT treatment $(n=591)$

\begin{tabular}{|c|c|c|c|c|c|c|}
\hline & \multicolumn{2}{|c|}{ Females } & \multicolumn{2}{|c|}{ Males } & \multicolumn{2}{|c|}{ Total } \\
\hline & $\mathrm{n}$ & Per cent & $\bar{n}$ & Per cent & $\bar{n}$ & Per cent \\
\hline Client non-adherence & 132 & 31.3 & 60 & 35.3 & 192 & 32.5 \\
\hline Referral by therapist & 21 & 5.0 & 18 & 10.6 & 39 & 6.6 \\
\hline Completed programme & 268 & 63.7 & 92 & 54.1 & 360 & 60.9 \\
\hline Total & 421 & 100.0 & 170 & 100.0 & 591 & 100.0 \\
\hline
\end{tabular}

decisions at the initial visit were also associated with a slightly increased risk of non-response.

Low $\chi^{2}$ values and high $\mathrm{p}$ values for the Hosmer and Lemeshow's goodness-of-fit test were observed (Nonadherence model $\chi^{2} 4.65$ and $\mathrm{p}=0.79$; Non-response model $\chi^{2} 9.16$ and $p=0.33$ ) suggesting that the models explain the variation in the response variables at a satisfactory level. No strong pair-wise linear correlations were found between the explanatory variables in the present data set.

\section{DISCUSSION}

The aim of this study was to assess the effects on anxiety, depression and psychological distress of a self-referral psychological service provided to young adults in a Swedish county and to explore client factors that predict non-adherence and non-response. In the analyses based on the ITT approach, we found a medium effect size with regard to psychological distress, whereas small effect sizes were observed with regard to anxiety and depression. When the analyses were restricted to clients who had completed an individually agreed treatment programme, a large effect size was observed with regard to psychological distress; medium effect sizes were observed with regard to anxiety and depression. The difference between the analytic approaches can be explained by the fact that about one-third of the clients discontinued the intervention before completing the programme. In particular, younger age and evaluating oneself as a worthless person at the visit were found to be risk factors for non-adherence; a high HADS-A score and reporting inability to concentrate were also found to be associated with discontinuation. Among the clients

Table 5 Multiple logistic regression model of non-adherence ( $n=552$; excluding clients subject to referral and self-management)

\begin{tabular}{llll}
\hline & OR & $\mathbf{9 0 \%} \mathbf{C l}$ & p Value \\
\hline Age & 0.845 & 0.796 to 0.897 & 0.0001 \\
Able to concentrate & 0.683 & 0.486 to 0.958 & 0.0641 \\
Thinking of oneself as a & 1.616 & 1.161 to 2.25 & 0.0169 \\
worthless person & & & \\
HADS-A & 1.045 & 1.004 to 1.087 & 0.0702 \\
\hline HADS-A, Hospital Anxiety and Depression Scale Anxiety.
\end{tabular}

who completed the intervention, being unhappy and depressed and not being able to enjoy normal day-to-day activities at their first visit were most strongly associated with persistent case status, in parallel with high initial scores on HADS-A and HADS-D.

The effect sizes observed among the clients who completed the CBT-based intervention (1.36 for anxiety and 1.48 for depression) were similar to those reported from pilot sites in the British IAPT programme (combined effect size 1.50) provided to adults of all ages. ${ }^{30}$ However, the ITT analysis of our data revealed considerably lower effect sizes $(0.58$ for anxiety and 0.56 for depression $)$, just as in the British programme (combined effect size in IAPT 0.60 ) and only $31 \%$ of clients admitted to the intervention recovered (compared with $24 \%$ in the IAPT programme). Thus, the high rate of non-adherence can explain a substantial part of the modest effect sizes and low proportion of recovered cases at the total client population level. Psychological treatment centres are expected to have a population-level impact on mental illness, ${ }^{4}$ therefore knowledge of the factors and mechanisms that contribute to non-adherence is of central importance. It has been reported that ethnic minority status, conduct problems, greater symptom severity and symptoms of attention-deficit/hyperactivity disorder (ADHD) increase the risk of treatment dropout among young people. ${ }^{13} 3132$ In our explorative analysis, we also found associations between treatment dropout, greater symptom severity and symptoms associated with ADHD, that is, between non-adherence, high initial-HADS-A

Table 6 Multiple logistic regression model of non-response to treatment $(n=360$; excluding clients who did not complete the CBT-based intervention)

\begin{tabular}{|c|c|c|c|}
\hline & OR & $90 \% \mathrm{Cl}$ & p Value \\
\hline Constantly under strain & 0.588 & 0.347 to 0.996 & 0.0971 \\
\hline $\begin{array}{l}\text { Able to enjoy normal } \\
\text { day-to-day activities }\end{array}$ & 0.37 & 0.219 to 0.625 & 0.0018 \\
\hline $\begin{array}{l}\text { Capable of making } \\
\text { decisions }\end{array}$ & 1.602 & 1.033 to 2.484 & 0.0772 \\
\hline Unhappy and depressed & 2.985 & 1.596 to 5.583 & 0.0041 \\
\hline HADS-A & 1.151 & 1.09 to 1.215 & 0.0001 \\
\hline HADS-D & 1.091 & 1.021 to 1.166 & 0.0305 \\
\hline
\end{tabular}


score, and an initial self-report of being unable to concentrate. Nonetheless, we also found that younger age and evaluating oneself as a worthless person at the first visit were the most prominent risk factors for nonadherence. It is possible that the latter factors reveal that some clients were not mature enough to engage in a therapeutic intervention on their own. Involvement in a psychological intervention requires a basic level of independence and life-structuring skills. Such an interpretation is also supported by a previous study from which personal-level reasons for dropout were reported to include miscommunication with the therapist and forgetting about appointments and homework tasks. ${ }^{12}{ }^{33}$ These findings suggest a need for innovative strategies to increase the engagement of young clients in treatment. It may also be possible to offer Internet therapy, group therapy and low-intensity or short treatment alternatives in a stepped-care model. In addition, administrative measures that could be introduced immediately include appointment reminder systems for cell phones, for example, based on short text messages. The high nonadherence rates also suggest a need to extend the collection of baseline data at psychological treatment centres for young people to include, for instance, data on sociodemographic variables and ethnicity.

Within the IAPT programme in the UK, self-referral has been tested to investigate if this route into the service facilitates access for groups who are not well served by general practitioner (GP) referral alone. ${ }^{6}$ Experiences suggest that opening up a psychological service to self-referral can improve access for ethnical minority groups and those with stigmatising diagnoses (such as social phobia and obsessive-compulsive disorder), while still not attracting clients with less severe problems than those referred. Also, self-referrers had clinical outcomes that were as good as GP referrals. Although the effects of self-referral not were explicitly evaluated for the Young Adults Centre, our experiences regarding problem severity are in agreement with those reported from the IAPT programme. Four out of five clients admitted to the programme scored above the case breakpoint for anxiety (HADS-A) and every second client scored above the case breakpoint for depression (HADS-D). In addition to clients with anxiety and depression, about $4 \%$ of the clients seen at the centre had symptoms of more serious psychiatric disease and could be provided with an early referral to specialist care. Thus, although cautiously, we infer that a selfreferral psychological treatment centre using a telephone triage system can attract and select an adequate population of young clients in need of their services. However, the present study did not evaluate whether the access to the services at the Young Adults Centre was equally distributed between groups of young people with different ethnic and sociodemographic backgrounds. From the headspace programme in Australia, client demographic characteristics tending to reflect population-level distributions have been reported, ${ }^{9}$ even though young people who were born outside Australia were underrepresented. Studies of whether the services at the Young Adults Centre are sufficiently accessible also for young people born abroad or not having Swedish as their first language are thus warranted.

This study has both strengths and weaknesses that need to be taken into account when interpreting the results. The strengths of the study include that it was performed in a routine setting and that the data set involved all clients admitted to the centre during the study period. Based on the underlying population perspective on the mental health of young people, the analyses were performed using an ITT approach to structuring of data. Evaluations using the ITT and completer approaches in parallel have recently been called for when reporting psychological interventions. ${ }^{30} 34$ Nonetheless, the study findings must also be interpreted in light of the limitations of observational implementation studies. Such study designs cannot ascertain what proportion of the recovery rates can be accounted for by factors such as spontaneous remission over time. It is known that clients with depression and anxiety may recover without professional help, implying that some clients included in the present study would have improved even without access to the services. In the absence of control groups and regulated conditions in routine settings, one has to rely on comparisons with other samples in order to decide whether a treatment programme is effective. However, if the observed improvements with treatment are considerably larger than those observed in samples of people who had no, or minimal, treatment, one can be fairly confident that the treatment was effective. The literature suggests that natural recovery varies with the previous duration of a psychological disorder. Several studies ${ }^{35}{ }^{36}$ have investigated recovery in recent onset cases of depression and/or anxiety in primary care and have reported recovery rates of $50-70 \%$ over the following few months in patients who received modest GP treatment as usual. In contrast, studies that have recruited cases with a previous duration of 6 months or more tend to report very low recovery rates in wait-list samples. For example, Posternak and Miller's ${ }^{37}$ meta-analysis of wait-list control groups reported that the average recovery rate from depression is approximately $20 \%$. In randomised controlled trials of CBT for anxiety disorders with a duration of at least 6 months, ${ }^{38-40}$ recovery rates rarely exceed $5 \%$ in the wait list. This implies that it is reasonable to assume that the evaluated treatment had an effect compared with no treatment. However, because no follow-up period was included in this study, no conclusions can be made regarding the persistence of the effect. A further limitation of this study is the lack of standardised diagnostic interviews. Although the selfreport measures, the HADS-A/D and GHQ-12, have solid psychometric properties and the cut points used are those reported as being the scores with the best balance of diagnostic specificity and sensitivity, they do 
not formally diagnose. Although it is likely that most of our clients would attract a formal diagnosis if interviewed by a diagnostician, no attempt was made to determine the exact diagnostic status of the client population. Another potential bias arises from the collection of selfevaluation data in the presence of the therapists. Some protection against bias is provided by the self-report method of data collection but the potential for the demands of this situation to influence the results remains. It also must be acknowledged that 'recovery' in this evaluation study was interpreted as 'absence of clinical symptoms', while the term generally in the mental health policy and service literature refers to 'living well with or without the symptoms of mental ill-health'. However, the latter interpretation of recovery was still used as the foundation for the clinical intervention programme at the Young Adults Center. ${ }^{25}$ In future evaluations of self-referral psychological services such as the Young Adults Center in routine settings, pre-post intervention measurement of variables associated to 'living well' is thus highly warranted, for example, days out of role and overall social functioning. ${ }^{9}$ Finally, it should be noted that the level of significance was set to $10 \%$ in the computations of the multiple logistic regression models of non-adherence and non-response to treatment. Based on that limited knowledge is available about factors associated with psychological treatment response and adherence in young adults, it was considered suitable to include a wide span of factors in the exploratory models. ${ }^{42}$ The findings are to be used to inform the design of further studies where more rigorous definitions of statistical significance are employed.

In this observational study of a self-referral psychological service provided to young adults, we found a medium effect size with regard to psychological distress; small effect sizes were observed with regard to anxiety and depression. However, when the analyses included only clients who completed the intervention programme, a large effect size was observed with regard to psychological distress; medium effect sizes were observed with regard to anxiety and depression. We conclude that provision of psychological services to young people through a self-referral centre has strong potential to improve the long-term mental health in communities, but non-adherence is a central challenge. In addition, several methodological issues associated with evaluations of services such as that presently investigated warrant further study, for example, the choice of primary end points and methods for calculating effect size.

Contributors KH, TT, PT, A-KA, LF and KD conceived the study. KH and TT designed the study. TS and $\mathrm{KJ}$ analysed the data. $\mathrm{KH}$ and TT drafted the manuscript. PT, A-KA, LF, TS, KJ and KD revised the manuscript providing intellectual content. TT is the guarantor of the final version.

Funding This study was supported by a research grant (LI0-446971) from Region Östergötland. The grant provider had no influence on the study design, performance or report.

Competing interests None declared.
Ethics approval This study is considered a routine service evaluation, that is, it was based on routine databases established for the purpose of systematically and continuously developing and securing the quality of psychological services. The study did therefore not fall under the definition of research according to Swedish legislation (SFS 2008:355) because we did not manipulate clinicians' treatment decisions or use experimental interventions. Accordingly, no approval from an ethics committee was required for the study. All clients were as a part of the routines at the clinic informed verbally and in writing about that their data may be used for analyses of service quality assurance and their verbal consent were asked for. Written consent was not requested due to that all clients were asked to fill in self-report forms for collection of diagnostic data. The same data were used for service quality control. Non-adherence with this procedure was noted in the electronic client record. No informed consent from the next of kin, caretakers, or guardians on behalf of the minors included in the service quality evaluation was obtained. Minors older than 15 years of age were in accordance with established practices in Swedish healthcare assumed to make own decisions regarding their care and the associated quality assessment. The age was determined by the upper age limit for paediatric care in Sweden. Personal identification data were removed from the records (all information was de-identified) prior to that the data set was received by the authors for statistical analyses. The evaluation design was confirmed by consultation with the Ethics Committee at Linköping University before initiation of the data collection.

Provenance and peer review Not commissioned; externally peer reviewed.

Data sharing statement No additional data are available.

Open Access This is an Open Access article distributed in accordance with the Creative Commons Attribution Non Commercial (CC BY-NC 4.0) license, which permits others to distribute, remix, adapt, build upon this work noncommercially, and license their derivative works on different terms, provided the original work is properly cited and the use is non-commercial. See: http:// creativecommons.org/licenses/by-nc/4.0/

\section{REFERENCES}

1. Vos T, Flaxman AD, Naghavi M, et al. Years lived with disability (YLDs) for 1160 sequelae of 289 diseases and injuries 1990-2010: a systematic analysis for the Global Burden of Disease Study 2010. Lancet 2012;380:2163-96.

2. Murray CJ, Vos T, Lozano R, et al. Disability-adjusted life years (DALYs) for 291 diseases and injuries in 21 regions, 1990-2010: a systematic analysis for the Global Burden of Disease Study 2010. Lancet 2012;380:2197-223.

3. Patel V, Flisher AJ, Hetrick S, et al. Mental health of young people: a global public-health challenge. Lancet 2007;369:1302-13.

4. Layard $\mathrm{R}$. The case for psychological treatment centres. $B M J$ 2006;332:1030-2.

5. Cahill J, Barkham M, Stiles WB. Systematic review of practice-based research on psychological therapies in routine clinic settings. $\mathrm{Br} \mathrm{J}$ Clin Psychol 2010;49:421-53.

6. Clark DM, Layard R, Smithies R, et al. Improving access to psychological therapy: initial evaluation of two UK demonstration sites. Behav Res Ther 2009;47:910-20.

7. Richards DA, Borglin G. Implementation of psychological therapies for anxiety and depression in routine practice: two year prospective cohort study. J Affect Disord 2011;133:51-60.

8. McGorry PD, Tanti C, Stokes R, et al. headspace: Australia's National Youth Mental Health Foundation-where young minds come first. Med J Aust 2007;187(7 Suppl):S68-70.

9. Rickwood DJ, Telford NR, Parker AG, et al. headspace-Australia's innovation in youth mental health: who are the clients and why are they presenting?. Med J Aust 2014;200:108-11.

10. Rickwood D, Van Dyke N, Telford N. Innovation in youth mental health services in Australia: common characteristics across the first headspace centres. Early Interv Psychiatry 2015;9:29-37.

11. Rickwood DJ, Telford N, Mazze K, et al. Changes in psychological distress and psychosocial functioning for young people accessing headspace centres for mental health problems. Med J Aust 2015;202:537-42.

12. Harrison ME, McKay MM, Bannon WM. Inner-city child mental health service use: the real question is why youth and families do not use services. Community Ment Health J 2004;40:119-31. 
13. Gopalan G, Goldstein L, Klingenstein K, et al. Engaging families into child mental health treatment: updates and special considerations. $J$ Can Acad Child Adolesc Psychiatry 2010;19:182-96.

14. Lefforge NL, Donohue B, Strada MJ. Improving session attendance in mental health and substance abuse settings: a review of controlled studies. Behav Ther 2007;38:1-22.

15. Wenemark M, Fornander L, Borgstedt-Risberg M. Östgötens psykiska hälsa. Om unga vuxna. Rapport 2006:4. Linköping Folkhälsovetenskapligt centrum. Landstinget i Östergötland, 2006.

16. Bremberg S. Ungdomar, stress och psykisk ohälsa. Stockholm: Statens offentliga utredningar, 2006.

17. Andersson G, Asmundson GJ, Carlbring P, et al. Is CBT already the dominant paradigm in psychotherapy research and practice? Cogn Behav Ther 2005;34:1-2.

18. Mineka S, Watson D, Clark LA. Comorbidity of anxiety and unipolar mood disorders. Annu Rev Psychol 1998;49:377-412.

19. Barlow DH. Anxiety and its disorders: the nature and treatment of anxiety and panic. 2nd edn. New York: Guilford Press, 2002.

20. Reardon JM, Williams NL. The specificity of cognitive vulnerabilities to emotional disorders: anxiety sensitivity, looming vulnerability and explanatory style. J Anxiety Disord 2007;21:625-43.

21. Dozois DJA, Dobson KS, eds. The prevention of anxiety and depression: theory, research, and practice. Washington DC: American Psychological Association, 2004.

22. McEvoy PM, Nathan P. Effectiveness of cognitive behavior therapy for diagnostically heterogeneous groups: a benchmarking study. $J$ Consult Clin Psychol 2007;75:344-50.

23. Barlow DH, ed. Clinical handbook of psychological disorders. A stepby-step treatment manual. New York: Guilford Publications, 2001.

24. O'Donohue WT, Fisher JE, Hayes SC. Cognitive behavior therapy: applying empirically supported techniques in your practice. New York: John Wiley \& Sons, 2004.

25. Roth-Ledley D, Marx BP, Heimberg RG. Making cognitive-behavioral therapy work. New York: The Guildford Press, 2005.

26. Tait RJ, French DJ, Hulse GK. Validity and psychometric properties of the General Health Questionnaire-12 in young Australian adolescents. Aust N Z J Psychiatry 2003;37:374-81.

27. Bjelland I, Dahl AA, Haug TT, et al. The validity of the Hospital Anxiety and Depression Scale. An updated literature review. $J$ Psychosom Res 2002;52:69-77.

28. Fritz CO, Morris PE, Richler JJ. Effect size estimates: current use, calculations, and interpretation. J Exp Psychol Gen 2012;141:2-18.

29. Bowerman B, O'Connell R, Koehler A. Forecasting, time series and regression (Vol. Fjärde). Ohio: Curt Hinrichs, 2005.
30. Barkham M, Stiles WB, Connell J, et al. Psychological treatment outcomes in routine NHS services: what do we mean by treatment effectiveness? Psychol Psychother 2012;85:1-16.

31. Baruch G, Vrouva I, Fearon P. A follow-up study of characteristics of young people that dropout and continue psychotherapy: service implications for a clinic in the community. Child Adolesc Ment Health 2009;14:69-75.

32. Johnson E, Mellor D, Bran P. Differences in dropout between diagnoses in child and adolescent mental health services. Clin Child Psychol Psychiatry 2008;13:515-30.

33. Barnes $M$, Sherlock $S$, Thomas $L$, et al. No pain, no gain: depressed clients' experiences of cognitive behavioural therapy. Br J Clin Psychol 2013;52:347-64

34. Delgadillo J, McMillan D, Leach C, et al. Benchmarking routine psychological services: a discussion of challenges and methods. Behav Cogn Psychother 2014;42:16-30.

35. Kendrick T, Simons L, Mynors-Wallis L, et al. Cost-effectiveness of referral for generic care or problem-solving treatment from community mental health nurses, compared with usual general practitioner care for common mental disorders: randomised controlled trial. Br J Psychiatry 2006;189:50-9.

36. Spijker J, de Graaf R, Bijl RV, et al. Duration of major depressive episodes in the general population: results from the Netherlands Mental Health Survey and Incidence Study (NEMESIS). $\mathrm{Br} J$ Psychiatry 2002;181:208-13.

37. Posternak MA, Miller I. Untreated short-term course of major depression: a meta-analysis of outcomes from studies using wait-list control groups. J Affect Disord 2001;66:139-46.

38. Clark DM, Salkovskis PM, Hackmann A, et al. Two psychological treatments for hypochondriasis: a randomized controlled trial. $\mathrm{Br} J$ Psychiatry 1998;173:218-25.

39. Clark DM, Ehlers A, Hackmann A, et al. Cognitive therapy and exposure plus applied relaxation in social phobia: a randomised controlled trial. J Consult Clin Psychol 2006;74:568-78.

40. Ehlers A, Clark DM, Hackmann A, et al. Cognitive therapy for post-traumatic stress disorder: development and evaluation. Behav Res Ther 2005;43:413-31.

41. Smith-Merry J, Freeman R, Sturdy S. Implementing recovery: an analysis of the key technologies in Scotland. Int $J$ Ment Health Syst 2011;5:11.

42. Poitevineau J, Lecoutre B. Interpretation of significance levels by psychological researchers: the .05 cliff effect may be overstated. Psychon Bull Rev 2001;8:847-50. 